\title{
Unveiling the Role of Atomically Dispersed Active Sites Over Amorphous Iron Oxide- Supported Pt Catalysts for Complete Catalytic Ozonation of Toluene at Low Temperature
}

Rui Liu ${ }^{a, 1}$, Shejiang Liu ${ }^{a}$, Hui Ding ${ }^{* a, c, ~ 1, ~ D a n ~ Z h a o ~}{ }^{a}$, Jianfeng Fu ${ }^{a}$, Yuxin Zhang ${ }^{b}$, Wangchen Huo ${ }^{b}$, Gang (Kevin) Li ${ }^{{ }^{*} c}$

a School of Environmental Science \& Engineering, Tianjin University, Jinnan District, Tianjin, 300350, China.

b State Key Laboratory of Mechanical Transmissions, College of Materials Science and Engineering, Chongqing University, Chongqing 400044, PR China.

c Department of Chemical and Biomolecular Engineering, The University of Melbourne, Melbourne, VIC 3010, Australia.

AUTHOR INFORMATION

*Corresponding Author

Tel.: +86-27404701; Fax: +86-27404705.

E-mail: dinghui@tju.edu.cn; li.g@unimelb.edu.au.

Supporting figures and tables. 


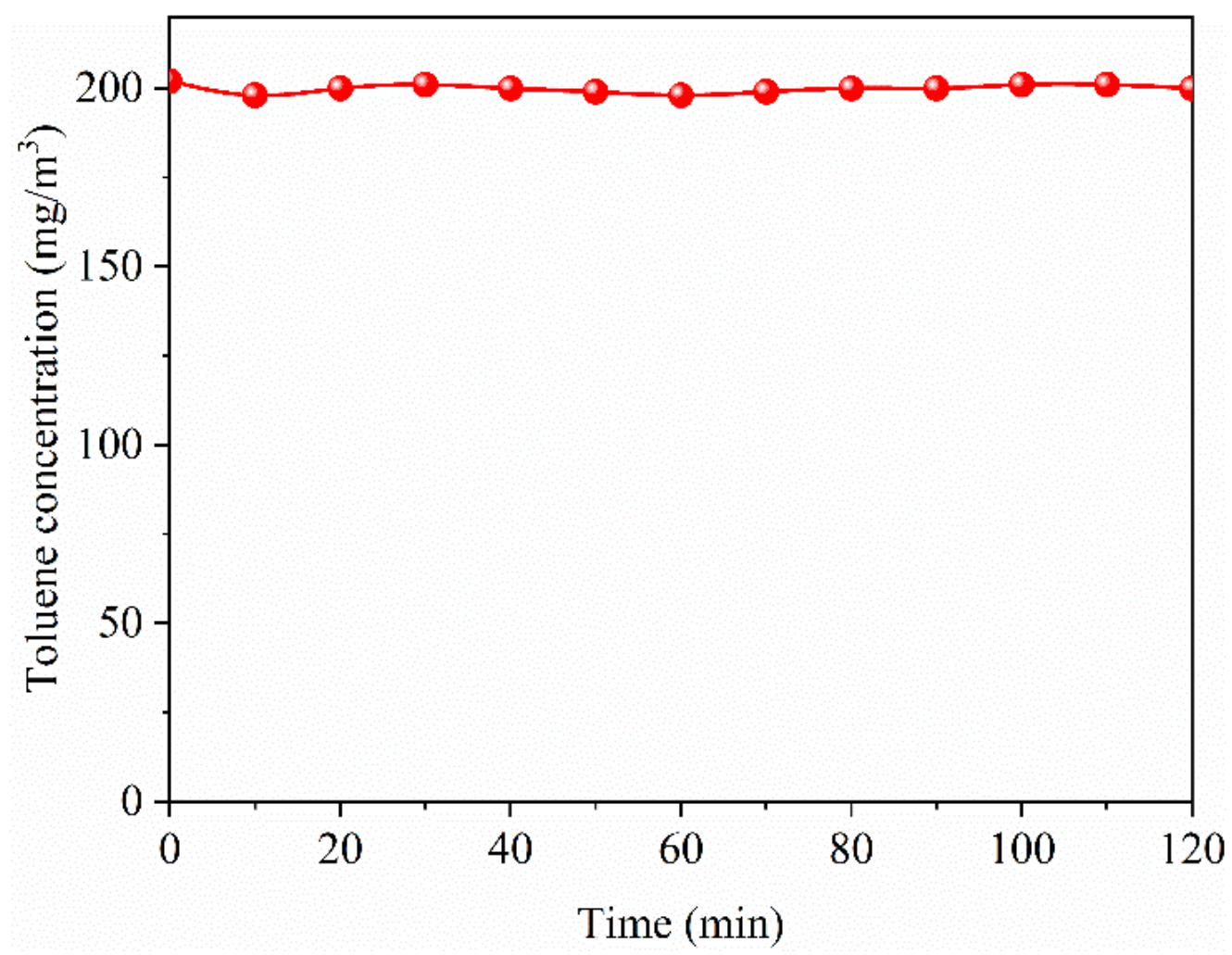

Figure S1. The blank experiment of $\mathrm{O}_{3}$ alone degradation toluene. 


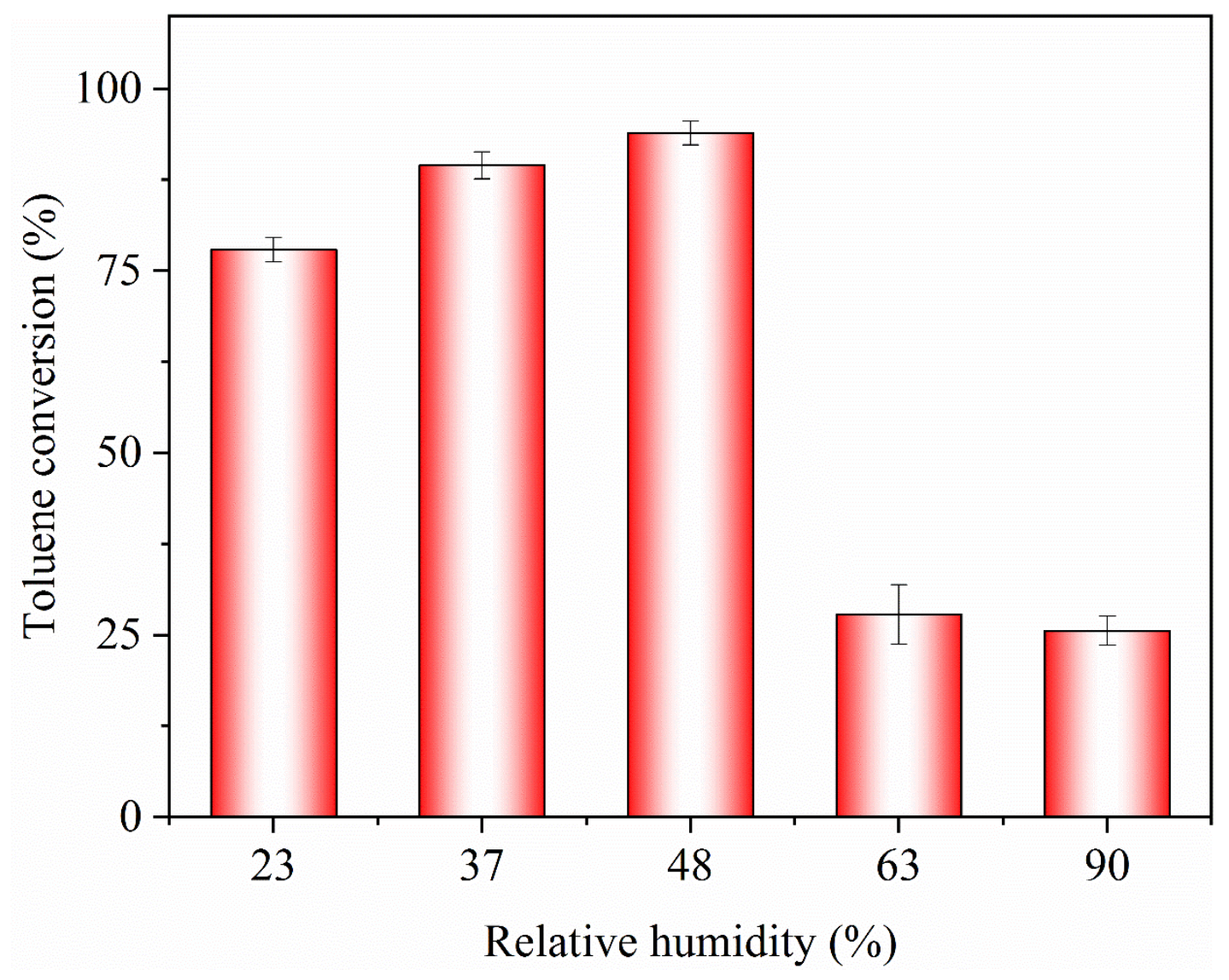

Figure S2. The effect of relative humidity on toluene degradation. 

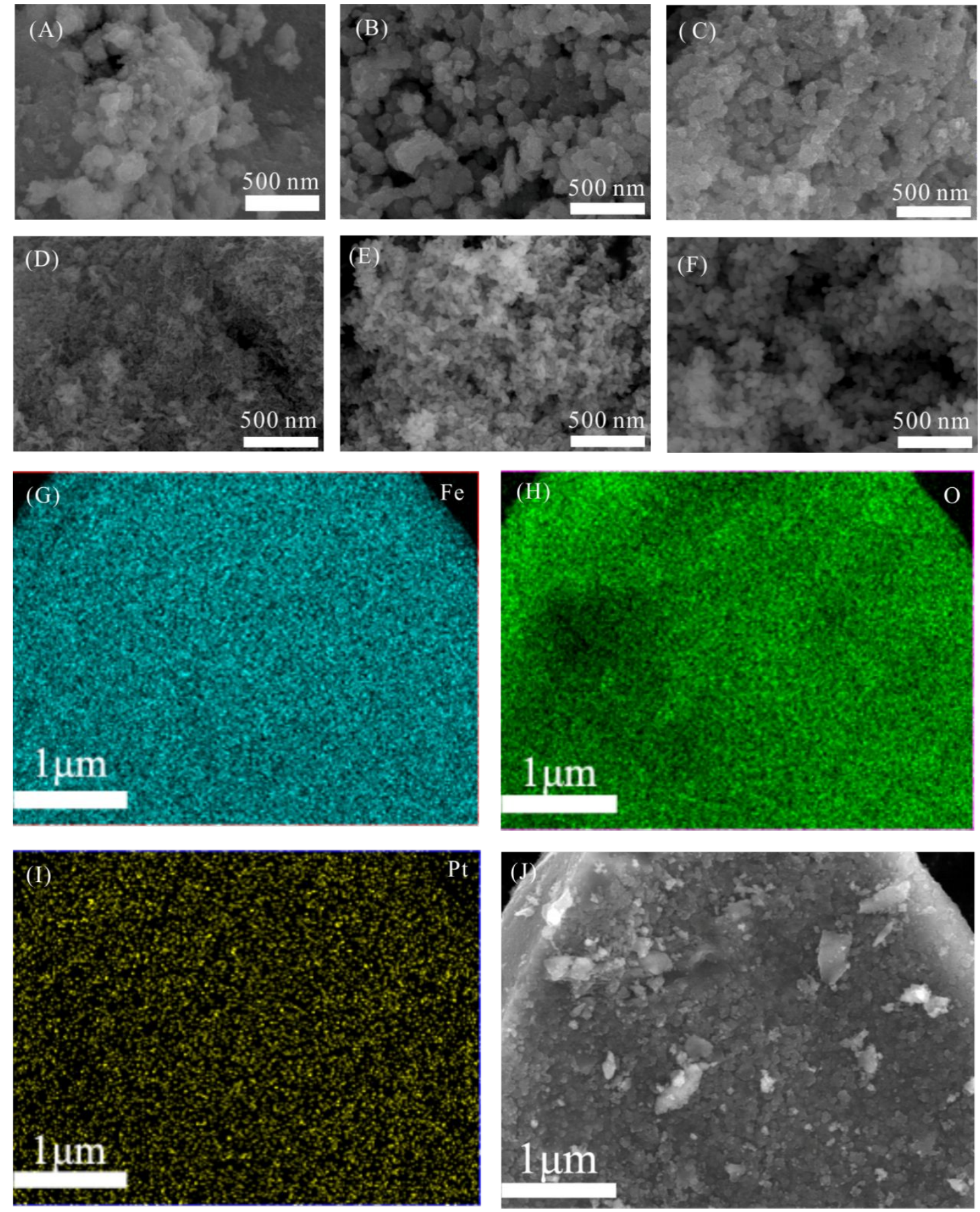

Figure S3. (A) (F) SEM images of Pt/FeOx-T catalysts $(\mathrm{T}=100,130,180,300,400$,

$\left.500{ }^{\circ} \mathrm{C}\right) ;(\mathrm{G}) \sim(\mathrm{J})$ EDS mapping images of $\mathrm{Pt} / \mathrm{FeOx}-180$ catalyst. 

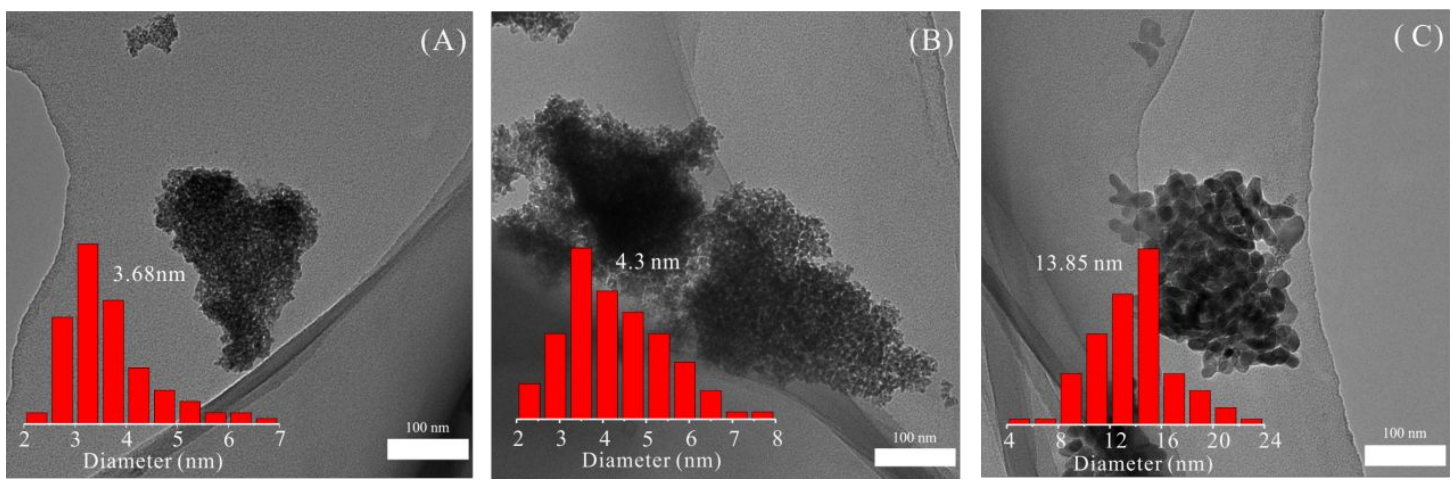

Figure S4. (A) (C) TEM images and particle diameter distributions of $\mathrm{Pt} / \mathrm{FeOx}-\mathrm{T}$ catalysts $\left(\mathrm{T}=100,180\right.$ and $\left.300^{\circ} \mathrm{C}\right)$. 

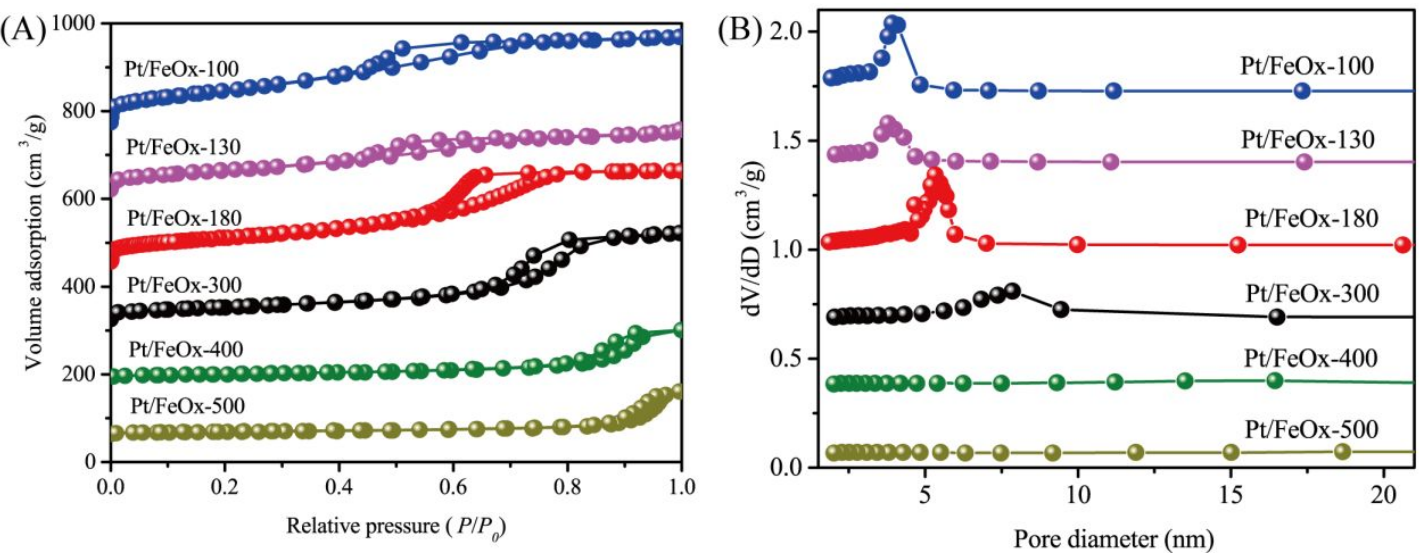

Figure S5. (A) Nitrogen adsorption-desorption isotherms and (B) pore-size distributions of the samples. 

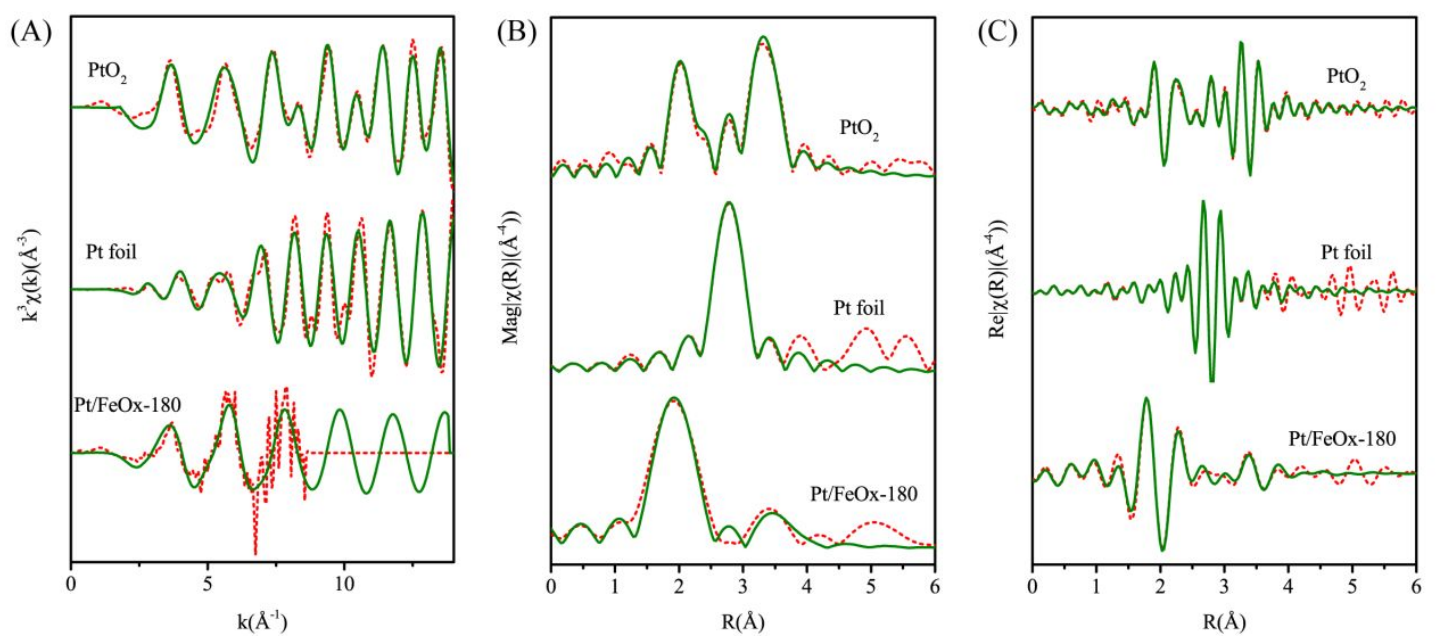

Figure S6. (A) $\mathrm{k}^{3}$-weighted chi spectra (red-dash line) and best fit (green line); (B)

Fourier-transformed magnitude spectra; (C) Fourier-transformed real part. 
Table S1. Comparison of performances for toluene oxidation over various Pt-based catalysts

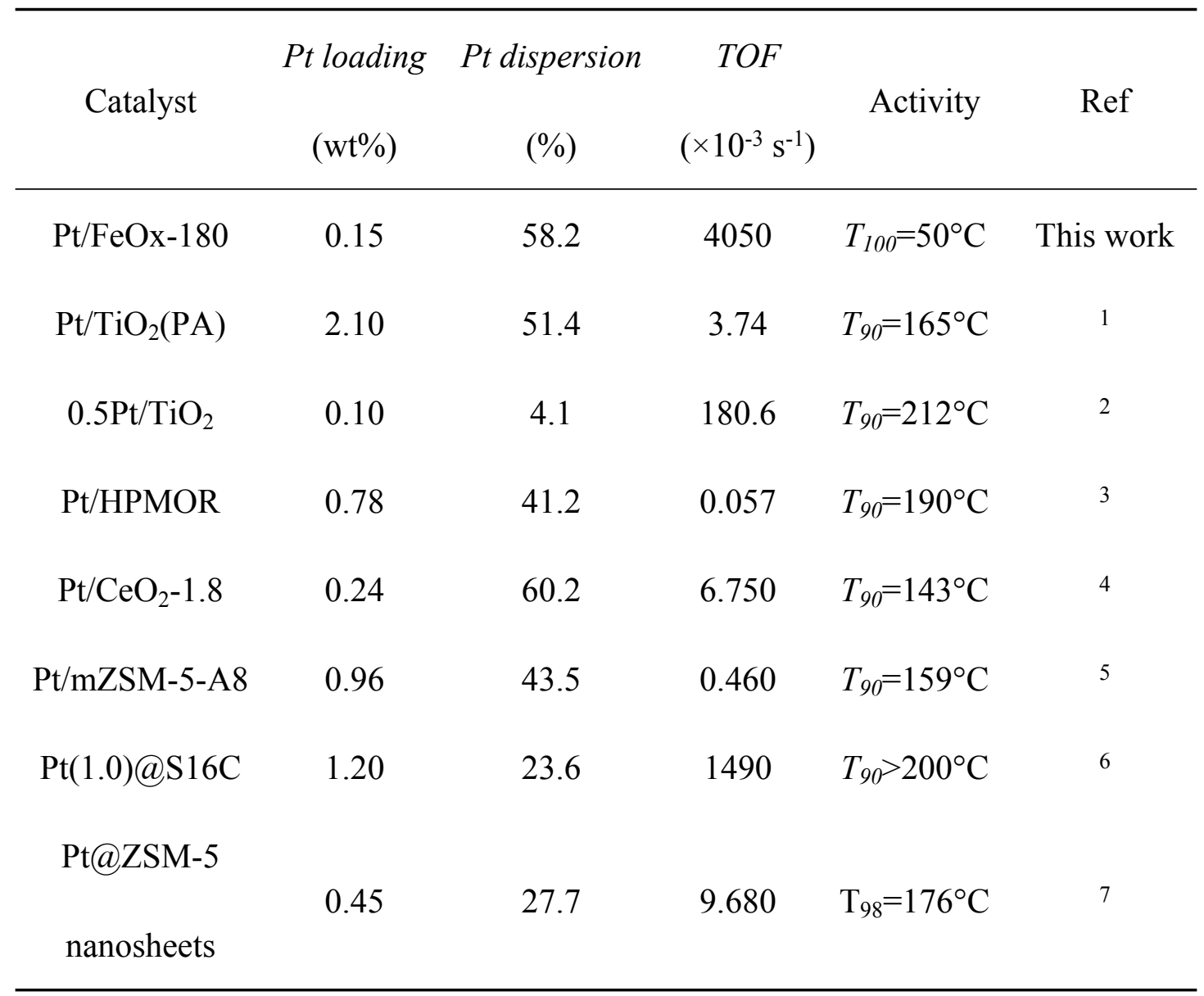


Table S2. Texture properties of $\mathrm{Pt} / \mathrm{FeOx}$ catalysts.

\begin{tabular}{cccc}
\hline Catalysts & $\mathrm{S}_{\mathrm{BET}}\left(\mathrm{m}^{2} \mathrm{~g}^{-1}\right)$ & Pore volume $\left(\mathrm{cm}^{3} \mathrm{~g}^{-1}\right)$ & Pore size $(\mathrm{nm})^{\mathrm{a}}$ \\
\hline $\mathrm{Pt} / \mathrm{FeOx}-100$ & 223 & 0.380 & 3.57 \\
$\mathrm{Pt} / \mathrm{FeOx}-130$ & 207 & 0.260 & 3.92 \\
$\mathrm{Pt} / \mathrm{FeOx}-180$ & 185 & 0.400 & 4.65 \\
$\mathrm{Pt} / \mathrm{FeOx}-300$ & 118 & 0.360 & 7.37 \\
$\mathrm{Pt} / \mathrm{FeOx}-400$ & 37 & 0.180 & 12.1 \\
$\mathrm{Pt} / \mathrm{FeOx}-500$ & 23 & 0.160 & 18.8 \\
\hline
\end{tabular}

a Average pore diameters calculated from desorption branches using BJH model. 
Table S3. The ratio of $\mathrm{Pt}^{4+} / \mathrm{Pt}^{2+} / \mathrm{Pt}^{0},-\mathrm{OH} / \mathrm{O}_{\text {latt }}$ and $\mathrm{Fe}^{3+} / \mathrm{Fe}^{2+}$ of $\mathrm{Pt} / \mathrm{FeOx}$ catalysts with different calcined temperatures.

\begin{tabular}{cccc}
\hline Catalyst & $\mathrm{Pt}^{4+} / \mathrm{Pt}^{2+} / \mathrm{Pt}^{0}$ & $-\mathrm{OH} / \mathrm{O}_{\text {latt }}$ & $\mathrm{Fe}^{3+} / \mathrm{Fe}^{2+}$ \\
\hline $\mathrm{Pt} / \mathrm{FeOx}-130$ & $0 / 0 / 1.03$ & 1.16 & 1.14 \\
$\mathrm{Pt} / \mathrm{FeOx}-180$ & $1.22 / 0.55 / 0$ & 3.35 & 0.68 \\
$\mathrm{Pt} / \mathrm{FeOx}-300$ & $0 / 1.12 / 0$ & 1.02 & 0.81 \\
$\mathrm{Pt} / \mathrm{FeOx}-400$ & $0 / 1.75 / 1.58$ & 0.44 & 0.76 \\
\hline
\end{tabular}


Table S4. Actual and theoretical $\mathrm{H}_{2}$ consumption of $\mathrm{Pt} / \mathrm{FeOx}-180$.

\begin{tabular}{cccc}
\hline Catalyst & Actual $\mathrm{H}_{2}$ & Theoretical $\mathrm{H}_{2}$ & Actual loading \\
& consumption & consumption & amount $/ \%$ \\
& amount $/ \mathrm{mmol} / \mathrm{g}$ & amount $/ \mathrm{mmol} / \mathrm{g}$ & \\
\hline $\mathrm{Pt} / \mathrm{FeOx}-180$ & 0.16 & $3.44 \times 10^{-6}$ & 0.15 \\
\hline
\end{tabular}

a Data were determined according to the ICP-AES. 
Table S5. Dada of selectivity calculation for toluene degradation over Pt/FeOx-180 catalyst at $50^{\circ} \mathrm{C}$.

\begin{tabular}{ccccc}
\hline & $\mathrm{CO}_{2} / \mathrm{ppm}$ & $\mathrm{CO} / \mathrm{ppm}$ & $\mathrm{CH}_{4} / \mathrm{ppm}$ & toluene/ppm \\
\hline $1^{\text {st } \text { test }}$ & 334.13 & 107.33 & 1.65 & 65.74 \\
$2^{\text {nd }}$ test & 333.62 & 105.24 & 0.62 & 65.49 \\
$3^{\text {rd }}$ test & 333.81 & 106.36 & 1.26 & 66.04 \\
average value & 333.85 & 106.31 & 1.17 & 65.76 \\
selectivity & 72.53 & 23.10 & 0.26 & \\
total carbon & & 95.88 & & \\
balance & & & & \\
\hline
\end{tabular}

a To calculate the selectivity easily, the ppm unit was used. 


\section{Reference:}

1. Lu, A. L.; Sun, H. L.; Zhang, N. W.; Che, L. M.; Shan, S. Y.; Luo, J.; Zheng, J. B.; Yang, L. F.; Peng, D. L.; Zhong, C. J.; Chen, B. H., Surface partial-charge-tuned enhancement of catalytic activity of platinum nanocatalysts for toluene oxidation. ACS Catal. 2019, 9, (8), 7431-7442.

2. Chen, X.; Zhao, Z. L.; Zhou, Y.; Zhu, Q. L.; Pan, Z. Y.; Lu, H. F., A facile route for spraying preparation of $\mathrm{Pt} / \mathrm{TiO}_{2}$ monolithic catalysts toward VOCs combustion. Appl. Catal. A-Gen. 2018, 566, 190-199.

3. Zhang, J. Y.; Rao, C.; Peng, H. G.; Peng, C.; Zhang, L.; Xu, X. L.; Liu, W. M.; Wang, Z.; Zhang, N.; Wang, X., Enhanced toluene combustion performance over Pt loaded hierarchical porous MOR zeolite. Chem. Eng. J. 2018, 334, 10-18.

4. Peng, R. S.; Li, S. J.; Sun, X. B.; Ren, Q. M.; Chen, L. M.; Fu, M. L.; Wu, J. L.; Ye, D. Q., Size effect of Pt nanoparticles on the catalytic oxidation of toluene over Pt/CeO 2 catalysts. Appl. Catal. B 2018, 220, 462-470.

5. Yang, D. Y.; Fu, S. Y.; Huang, S. S.; Deng, W.; Wang, Y.; Guo, L. M.; Ishihara, T., The preparation of hierarchical Pt/ZSM-5 catalysts and their performance for toluene catalytic combustion. Microporous Mesoporous Mater. 2020, 296, 109802109809.

6. Wu, H.-C.; Chen, T.-C.; Budi, C. S.; Huang, P.-H.; Chen, C.-S.; Kao, H.-M., Confinement of Pt nanoparticles in cage-type mesoporous silica SBA-16 as efficient catalysts for toluene oxidation: the effect of carboxylic groups on the mesopore surface. Catal. Sci. Technol. 2019, 9, (24), 6852-6862. 
7. Liu, G. Z.; Tian, Y. J.; Zhang, B. F.; Wang, L.; Zhang, X. W., Catalytic combustion of VOC on sandwich-structured Pt@ZSM-5 nanosheets prepared by controllable intercalation. J. Hazard. Mater. 2019, 367, 568-576. 